splitting of energy levels. But highprecision atomic and nuclear spectroscopic measurements set stringent upper limits on such effects.

Consider, for example, the case of the $2 S_{1 / 2} \rightarrow 3 P_{1 / 2}$ transition in the hydrogen atom. For simplicity, it will be discussed using semi-classical theory in which $r_{\text {eq }} \omega=$ $l /(m\langle r))$ where $m$ is the inertial mass of the electron and $\langle r\rangle=n^{2} a_{0}$ is the Bohr radius ( $n$ is the principal quantum number and $a_{0}$ $=0.53 \times 10^{-8} \mathrm{~cm}$ ). If equation (1) applies at the microscopic level, the electron gyroscope has

$$
\left|\frac{\Delta m_{\mathrm{R}}}{m}\right|=\kappa \frac{l}{m n^{2} a_{0} g} \sim 4.5 \frac{\hbar}{n^{2}}
$$

and one would expect the $2 S_{1 / 2} \rightarrow 3 P_{1 / 2}$ transition to be split by an energy $\Delta E=1.5$ $\mathrm{eV} \Delta m_{R} / m \approx 0.76 \mathrm{eV}$ due to the anomalous inertial mass of one of the magnetic substates of the $3 P$ level. This transition has been studied ${ }^{5}$ with a resolution better than the natural linewidth of $\approx 2 \times 10^{-6}$ $\mathrm{eV}$, and no anomalous splittings were observed. Very widely split components of the $3 \rightarrow 2$ transition can be excluded by the fact that the transition rates agree with conventional theory and are inconsistent with the notion that one component of the line is highly anomalous. Thus the upper limit on $\kappa$ from the electron gyroscope is roughly $4 \times 10^{5}$ times smaller than the value claimed by Hayasaka and Takeuchi.

A considerably more stringent limit comes from nuclear gyroscopes. The Mössbauer effect, for example in ${ }^{57} \mathrm{Fe}$, cleanly resolves all the hyperfine states corresponding to the allowed orientations of the nuclear angular momentum (see ref. 6 , for example). If equation (1) applies, one can estimate $\Delta m_{R} / m \approx 8.6$, where $l=\hbar$ amd $\langle r\rangle \approx 5$ fm for the $2 p$ neutron that 'jumps' in the Mössbauer transition. The energy splitting of the $14-\mathrm{keV} \gamma$-ray due to the splitting of the neutron inertial mass would be roughly

$\Delta E \approx 30 \mathrm{MeV} \frac{\Delta m_{\mathrm{R}}}{m} \approx 2.6 \times 10^{8} \mathrm{eV}$

It is known that there are no anomalous splittings in the ${ }^{57} \mathrm{Fe}$ transition greater than the natural linewidth of $\Delta E \approx 4.6 \times 10^{-9}$ $\mathrm{eV}$. This is nearly 17 orders of magnitude below the value one expects if Hayasaka's and Takeuchi's effect applied in the microscopic realm.

Physics Department, University of Washington,

Seattle 98198, USA

1. Hayasaka, H. \& Takeuchi, S. Phys. Rev. Lett. 63, 2701 (1989).

2. Faller, J.E., Hollander, W.J., Nelson, P.G. \& McHugh, M.P. Phys. Rev. Lett. 64, 825 (1990)

3. Quinn, T.J. \& Picard, A, Nature 343, 732 (1990).

3. Quinn, T.J. \& Picard, A, Nature 343, 732 (1990).

4. Heckel, B.R. et al. Phys. Rev. Lett. 63, 2705 (1989).
5. Goldsmith, J.E.M., Weber, E.W. \& Hänsch, T.W. Phys. Rev.

Lett. 41, 1525 (1978).

6. Violet, C.E. \& Pipkorn, D.N. J. appl. Phys. 42, 4339 (1971).

NATURE $\cdot$ VOL $345 \cdot 10$ MAY 1990

\title{
Radiation hazards and leukaemia
}

SIR-The articles by M. J. Gardner et al. (Bri. med. J. 300, 423-429 and 429-434; 1990 ), on the possible linkage between high exposure to radiation of fathers working at Sellafield and leukaemia clusters in their offspring, highlight the need for more careful monitoring of links between both occupational radiation and high natural radiation exposure, and disease in families of certain groups.

Gardner et al. suggest that the lack of a relationship between massive doses of radiation in survivors of the Hiroshima atomic-bomb explosion and leukaemia in their children may be because the genetic linkage requires a cumulative effect of smaller radiation doses over a long period. If such a linkage is possible, then it is surely imperative to study families where one or both parents may have been exposed to either of these types of radiation before conception, and where a child develops leukaemia. In this respect, data are required on families of various groups: reprocessing-plant and reactor workers, tin miners in southwest England, airline pilots with high exposure to cosmic rays, and those exposed to high natural radiation from radon, for example.

In the case of natural radiation exposure, for example, R. H. Clarke and T. R. E. Southwood (Nature 338, 197; 1989) pointed out that in parts of Cornwall in southwest England individual exposure levels to the $\alpha$-emitter radon and its daughters can rise to doses greater than 20 millisieverts $(\mathrm{mSv})$ a year, a high proportion of which comes from indoor radiation. The average individual exposure to radon in Cornwall in 1988 was given as 7.8 $\mathrm{mSv}$ a year compared with the national average of $2.5 \mathrm{mSv}$. This compares with average occupational exposure rates in the same period for workers in reactor plants of less than $1 \mathrm{mSv}$ a year and of about $5 \mathrm{mSv}$ for workers in reprocessing plants. Although no data were given for exposure to cosmic rays of airline pilots, Clarke and Southwood suggested that airline travellers received slightly greater annual exposure to cosmic rays than the average for individuals in the United Kingdom as a whole. The figure of greater than $20 \mathrm{mSv}$ a year for some people in southwest England can also be compared with the data of Gardner et al. for the four Sellafield fathers with children who developed leukaemia; the four had average exposures over several years of 14.6, 27, 27 and $7.5 \mathrm{mSv}$, respectively.

Given the kind of data available on indoor exposure to natural radiation in parts of Cornwall and elsewhere, what data do we have on leukaemia among children where the mothers have been exposed before pregnancy to high levels of radiation over comparable periods of time? If such data are available, how do they compare with data for workers in other high-exposure industries? Information of this kind is clearly important if genetic linkages between disease, and exposure of a parent to high natural or occupational radiation, are to be substantiated.

Department of Geology,

University of Exeter,

Exeter EX4 4QE, UK

\section{Protein instruction}

SIR-Townsend et al. ${ }^{\prime}$ recently reported the unexpected influence of peptides on the expression of complexes of class I MHC heavy chain with $\beta_{2}$-microglobulin $\left(\beta_{2} \mathrm{~m}\right)$ and, in that context, Parham ${ }^{2}$ defended a surprising instructional theory of protein folding. Attractive as they may appear, we should remember that such theories have vanished from immunology because they do not make much sense at the molecular level.

In water, the folding of large proteins is driven by large entropic terms arising from the need that their hydrophobic cores should be hidden ${ }^{3}$. Thus, folding is a spontaneous process, faster than chain synthesis. In contrast, protein-protein interactions are mediated by short-range low energy forces (electrostatic, hydrogen bonds, dispersion). Thus, stable complexes require the cooperation of many individual interatomic interactions.

It follows that two protein molecules will assemble together only if their overall shapes and force fields are already quite complementary before collision. Indeed, conformational changes might further stabilize the initial complex, but a better than random affinity must pre-exist the actual collision of the molecules: there is induced fit, not instructed folding.

The case of the peptide/class I MHC interaction raises even more difficulties. First an isolated peptide fluctuates among a large number of flexible conformers, so that its "instructive" value must be poor. Second, the numerous class I amino-acids in a position to bind the peptide in the stable complex are interspersed throughout the polypeptide chain (at locations $5,7,9,26,59,63,66,70,73$, $95,97,99,114,116,152,156,163$ - see refs 4,5 ) in such a way that no local interaction with the ligand could occur from an appreciably unfolded conformation.

Indeed, the results of Townsend $e t$ al. can still be interpreted within the standard, selectionist framework. In solution, the class I MHC heavy chains are dynamically distributed among a number of conformations of similar energy and topology, some of them with an appreciable affinity for a given peptide epitope. As a collision 